\title{
Preparation, characterization, and in vitro and in vivo investigation of chitosan-coated poly (d,l-lactide-co-glycolide) nanoparticles for intestinal delivery of exendin-4
}

\author{
This article was published in the following Dove Press journal: \\ International Journal of Nanomedicine \\ 18 March 2013 \\ Number of times this article has been viewed
}

\section{Mengshu Wangl,* \\ Yong Zhang ${ }^{1, *}$ \\ Jiao Feng' \\ Tiejun $\mathrm{Gu}^{\prime}$ \\ Qingguang Dong' \\ Xu Yang ${ }^{2}$ \\ Yanan Sun' \\ Yongge $\mathrm{Wu}^{\prime}$ \\ Yan Chen' \\ Wei Kong'}

'National Engineering Laboratory for AIDS Vaccine, College of Life Science, Jilin University, Changchun, People's Republic of China; ${ }^{2} \mathrm{BCHT}$ Biopharm Co, Ltd, Changchun, People's Republic of China

*These authors contributed equally to this work
Correspondence: Yan Chen National Engineering Laboratory for AIDS vaccine, College of Life Science, Jilin University, Changchun I300I2, People's Republic of China

Tel +86 43। 85I6 775 I

Fax +86 43I 85I6 7674

Email chen_yan@jlu.edu.cn

Wei Kong

Tel +86 43I 85I67823

Fax +86 43I 85I67674

Email weikong@jlu.edu.cn
Background: Exendin-4 is an incretin mimetic agent approved for type 2 diabetes treatment. However, the required frequent injections restrict its clinical application. Here, the potential use of chitosan-coated poly (d,1-lactide-co-glycolide) (CS-PLGA) nanoparticles was investigated for intestinal delivery of exendin-4.

Methods and results: Nanoparticles were prepared using a modified water-oil-water (w/o/w) emulsion solvent-evaporation method, followed by coating with chitosan. The physical properties, particle size, and cell toxicity of the nanoparticles were examined. The cellular uptake mechanism and transmembrane permeability were performed in Madin-Darby canine kidney-cell monolayers. Furthermore, in vivo intraduodenal administration of exendin-4-loaded nanoparticles was carried out in rats. The PLGA nanoparticle coating with chitosan led to a significant change in zeta potential, from negative to positive, accompanied by an increase in particle size of $\sim 30 \mathrm{~nm}$. Increases in both the molecular weight and degree of deacetylation of chitosan resulted in an observable increase in zeta potential but no apparent change in the particle size of $\sim 300 \mathrm{~nm}$. Both unmodified PLGA and chitosan-coated nanoparticles showed only slight cytotoxicity. Use of different temperatures and energy depletion suggested that the cellular uptake of both types of nanoparticles was energy-dependent. Further investigation revealed that the uptake of PLGA nanoparticles occurred via caveolin-mediated endocytosis and that of CS-PLGA nanoparticles involved both macropinocytosis and clathrin-mediated endocytosis, as evidenced by using endocytic inhibitors. However, under all conditions, CS-PLGA nanoparticles showed a greater potential to be transported into cells, as shown by flow cytometry and confocal microscopy. Transmembrane permeability analysis showed that unmodified and modified PLGA nanoparticles could improve the transport of exendin-4 by up to 8.9 - and 16.5-fold, respectively, consistent with the evaluation in rats.

Conclusion: The chitosan-coated nanoparticles have a higher transport potential over both free drug and unmodified particles, providing support for their potential development as a candidate oral delivery agent for exendin-4.

Keywords: chitosan, PLGA, intestinal absorption

\section{Introduction}

Exendin-4, an injectable glucagon-like peptide-1 (GLP-1) receptor agonist, was approved by the US Food and Drug Administration (FDA) for the treatment of type 2 diabetes mellitus in 2005. More recently, exendin-4 extended-release microspheres, the first once-weekly treatment for type 2 diabetes was approved by the FDA, in January 2012. ${ }^{1}$ However, the subcutaneous injection formulation limits 
its therapeutic application, due to reduced compliance related to both the expense associated with injection and pain at the site of injection. Therefore, alternative routes of administration for this drug, especially the oral dosage form have been the focus of attention for many researchers. However, oral delivery of peptides and proteins is restricted due to the excessive gastrointestinal enzymatic degradation and poor penetration of drugs across the intestinal membrane. ${ }^{2,3}$ Our previous study ${ }^{4}$ investigated the potential of exendin- 4 to be transported across the Madin-Darby canine kidney (MDCK) cell monolayer in the absence or presence of absorption enhancers. We found that exendin-4 alone is transported across the MDCK cell monolayer mainly by the passive paracellular pathway and that its transport capacity is marginal, with an apparent permeability coefficient of $1 \times 10^{-7} \mathrm{~cm} / \mathrm{s}$. In agreement with our previous work, another study found the intraduodenal bioavailability of exendin- 4 in rats, relative to its intravenous counterpart, to be $0.0053 \%$ and therefore concluded that the intestinal tract has limited potential as a route for exendin-4 administration. ${ }^{5}$ However, our previous study also demonstrated that the addition of absorption enhancers and enzyme inhibitors, such as chitosan and ethylenediaminetetraacetic acid (EDTA), could considerably increase transport of exendin-4 by 2.2- to 11.9-fold, without apparent cytotoxicity, by opening cellular tight junctions. ${ }^{4}$

Besides the coadministration of absorption enhancers and/or enzyme inhibitors, many other strategies have been developed to achieve the oral delivery of exendin-4, such as use of site-specific biotinylation, PEGylation (the attachment of polyethylene glycol [PEG] polymer chains), and bioencapsulation within plant cells. ${ }^{6-8}$ Although many improvements have been reported by these studies, the colloidal carrier systems, such as biodegradable and biocompatible nanoparticles, seem to be more promising due to their better safety, and controlled and tailored properties. It has been reported that use of $\mathrm{pH}$-sensitive nanoparticles fabricated with chitosan can significantly improve the oral bioavailability of exendin-4 in rats, relative to its subcutaneous counterpart, by up to $14.0 \% \pm 1.8 \% .{ }^{9}$ The significant improvement in bioavailability was attributed to the protection of drugs from enzymatic degradation and the increased permeation of drugs via the paracellular pathway (by opened tight junctions between epithelial cells), both mediated by chitosan.

Poly (d,1-lactide-co-glycolide) (PLGA) is one of the most widely used of the biodegradable and biocompatible nanoparticle polymers that have been approved by the FDA for drug delivery. PLGA nanoparticles have been used to protect drugs from enzymatic and chemical degradation, in order to improve therapeutic effects and target delivery. However, the slightly negative surface charge of PLGA nanoparticles tends to limit their interaction with the negatively charged cell surface and leads to lower intracellular uptake. Generally, cationic particles can easily interact with the negatively charged cell membrane and have improved cellular uptake. It has been reported that cationic polymers, such as polyethyleneimine and poly-L-lysine hydrobromide, can be used as candidate materials to modify PLGA nanoparticles for gene or drug delivery. ${ }^{10-13}$ Among these, chitosan seems to be the most suitable adjuvant due to its biodegradable and biocompatible, mucoadhesive, and permeability-enhancing properties. ${ }^{14,15}$ Mucus adhesion can prolong the duration of interaction between drugs and cells, while increased tissue permeability can allow dissemination of drugs through the paracellular transport pathway, via the opening of tight junctions between epithelial cells. Considering the great potential advantages of the use of chitosan-coated PLGA (CS-PLGA) nanoparticles as carriers, numerous studies have investigated their transport potential in gene, drug, and vaccine delivery, via various administration routes. ${ }^{16-22}$ However, to our knowledge, no study has yet investigated the potential of CS-PLGA nanoparticles for intestinal delivery of exendin-4 or reported the effect of molecular weight and degree of deacetylation on the characteristics of CS-PLGA nanoparticles.

In this study, PLGA nanoparticles were modified with chitosan of various molecular weights and degrees of deacetylation. The physicochemical properties, cytotoxicity, transport mechanism, and absorption-enhancing potential of the resultant CS-PLGA nanoparticles were characterized in the MDCK cell monolayer model. Furthermore, the efficiency of the intestinal delivery of exendin- 4 by optimized CS-PLGA nanoparticles was also investigated in rats.

\section{Materials and methods Materials}

Exendin-4 was purchased from BCHT Biopharm Co., Ltd. (Changchun, People's Republic of China). PLGA (lactide: glycolide $=50: 50$ ) was purchased from Durect Corporation (Birmingham, AL, USA). Chitosan (molecular weight: 200,000-400,000; degree of deacetylation: $85 \%$ and 95\%) was purchased from AK Biotech Co., Ltd. (Jinan City, People's Republic of China). Chlorpromazine, amiloride, filipin, 3-(2-benzothiazolyl)-7-(diethylamine)coumarin (6-coumarin), and 3-(4,5-dimethylthiazol-2-yl)-2,5-diphenyltetrazolium bromide (MTT) were purchased from SigmaAldrich (St Louis, MO, USA). Dulbecco's modified Eagle's medium (DMEM), fetal bovine serum (FBS), nonessential 
amino acids solution, penicillin-streptomycin solution, and trypsin solution were obtained from Life Technologies (Carlsbad, CA, USA). Millicell ${ }^{\circledR}$ cell culture inserts (24-well, $0.6 \mathrm{~cm}^{2}$ and $0.1 \mu \mathrm{m}$ ) were purchased from EMD Millipore Corp (Billerica, MA, USA), and 24-well Costar ${ }^{\circledR}$ cluster trays were purchased from Corning Inc (Corning, NY, USA). 1,1'-dioctadecyl-3,3,3',3'-tetramethylindocarbocyanine perchlorate (DiI) was from Life Technologies. All other chemical products were commercially available and of analytical grade.

\section{Nanoparticle preparation}

PLGA nanoparticles containing exendin-4 or 6-coumarin were prepared using a water-oil-water (w/o/w) emulsion solvent evaporation method, with slight modifications. ${ }^{23}$ Briefly, $1 \mathrm{mg}$ of exendin- 4 was dissolved in $200 \mu \mathrm{L}$ distilled water. The aqueous solution was emulsified with $1 \mathrm{~mL}$ dichloromethane containing PLGA, using a MICCRA D-1 homogenizer (ART Prozess- \& Labortechnik GmBH \& Co KG, Müllheim, Germany) at 20,000 rpm for 180 seconds. The primary emulsion was then added to $5 \mathrm{~mL}$ of $3 \%$ polyvinyl alcohol (PVA) and sonicated for 60 seconds to form a double emulsion, using a Vibra-Cell ${ }^{\mathrm{TM}}$ sonifier (VCX750; Sonics and Materials Inc, Newtown, CT, USA). The resulting emulsion was combined with $50 \mathrm{~mL}$ of $0.5 \%$ PVA and stirred for 3 hours at room temperature, allowing the dichloromethane to evaporate. The resulting PLGA nanoparticles were washed three times in distilled water, by centrifugation at 10,000 $\times \mathrm{g}($ Centrifuge 5810R; Eppendorf AG, Hamburg, Germany). The 6-coumarin-loaded nanoparticles were prepared under the same conditions as above except that $0.2 \mathrm{mg}$ of 6-coumarin was added to dichloromethane containing PLGA, with no drugs within the inner phase. To prepare the CS-PLGA nanoparticles, PLGA nanoparticles were added to the chitosan sodium acetate buffer ( $\mathrm{pH} 4.5,0-2.0 \mathrm{mg} / \mathrm{mL}$ ), under agitation with a magnetic stirrer. Subsequently, the mixture was centrifuged at $10,000 \times \mathrm{g}$ for 20 minutes, and the supernatant was decanted. Chitosans of different molecular weights and degrees of deacetylation were used, in order to investigate the effects of these physical properties on the particle size and zeta potential of CS-PLGA nanoparticles.

\section{Nanoparticle characterization}

The particle size and size distribution of nanoparticles in an aqueous environment were investigated by measuring dynamic light scattering (DLS), using the Zetasizer Nano ZS90 (Malvern Instruments, Malvern, UK), equipped with a helium-neon laser (633 nm) and 90-degree collecting optics.
In addition, zeta potentials were determined, based on the electrophoretic mobility of the nanoparticles in aqueous medium, with the same instrument.

\section{MDCK cell culture}

The MDCK cell line was obtained from the Cell Bank of Type Culture Collection of the Chinese Academy of Sciences (Shanghai Institute of Cell Biology, Shanghai, People's Republic of China). Cells were grown in DMEM supplemented with 10\% FBS, 1\% nonessential amino acids solution, and $1 \%$ penicillin-streptomycin and cultured in a humidified incubator at $37^{\circ} \mathrm{C}$ with $5 \% \mathrm{CO}_{2}$. The cells were allowed to grow to confluency and then trypsinized with $0.25 \%$ trypsin containing $0.02 \%$ EDTA, followed by seeding in a plate or Millicell culture insert for each experiment. Experiments were performed with cells, following 70-85 passages.

\section{Cytotoxicity studies}

MDCK cells were transferred to 96-well plates (Corning) at a density of $5 \times 10^{3}$ cells/well and cultured for 24 hours in a humidified incubator, at $37^{\circ} \mathrm{C}$ with $5 \% \mathrm{CO}_{2}$. The culture medium was then replaced with PLGA or CS-PLGA nanoparticles containing exendin-4 and diluted with culture medium to a concentration of $1.0,3.0$, or $5.0 \mathrm{mg} / \mathrm{mL}$. After 2 hours coincubation at $37^{\circ} \mathrm{C}$, the nanoparticles were replaced with $10 \mu \mathrm{L}$ of MTT $(5 \mathrm{mg} / \mathrm{mL}$ in Hank's balanced salt solution [HBSS]) and $90 \mu \mathrm{L}$ of incubation medium and incubated for a further 4 hours at $37^{\circ} \mathrm{C}$. After incubation, the medium was removed, and $100 \mu \mathrm{L}$ of dimethyl sulfoxide (DMSO) was added to the residual precipitates. The absorbance of the resulting DMSO solution was determined at $590 \mathrm{~nm}$, using an iMark $^{\mathrm{TM}}$ microplate reader (Bio-Rad Laboratories, Hercules, CA, USA). Cell viability was expressed as a percentage of the absorbance relative to that of the control. Control cells were not exposed to any materials. The experiments were performed with five replicate wells for each sample and control.

\section{Qualitative and quantitative analysis of the interaction between nanoparticles and MDCK cells \\ Effect of concentration and incubation time of nanoparticles on cellular uptake}

In order to investigate the effects of concentration of nanoparticles and coincubation time on cellular uptake, the MDCK cells were seeded in 96-well plates, at a density of $5 \times 10^{3}$ cells/well. After 3 days, the cells were washed and 
replaced with a series of nanoparticles containing 6-coumarin on the cell monolayer, at $37^{\circ} \mathrm{C}$. At specified incubation times, the cells were washed with ice-cold phosphate-buffered saline (PBS), and then, the amounts of nanoparticles taken up by the MDCK cells were measured using the Fluoroskan Ascent FL microplate reader (Thermo Fisher Scientific, Waltham, MA, USA). In addition, in order to eliminate the possible false-positive fluorescence resulting from free 6-coumarin, the equivalent amounts of free 6-coumarin released from the PLGA and CS-PLGA nanoparticles, respectively, were also subjected to the same experimental determination.

\section{Confocal laser scanning microscopic} and flow cytometric analysis

MDCK cells were grown on Fisherbrand microscope cover glass (Thermo Fisher Scientific), at a density of $3 \times 10^{5}$ cells/ well. When the cells were confluent, the culture medium was replaced with PLGA or CS-PLGA nanoparticles containing 6-coumarin $(0.2 \mathrm{mg} / \mathrm{mL})$, at $37^{\circ} \mathrm{C}$ for 2 hours. The cells were then washed five times with ice-cold PBS, fixed with 4\% paraformaldehyde for 30 minutes, and then counterstained with $5 \mu \mathrm{M}$ DiI for 20 minutes. Thereafter, the stained cells were observed using a Carl Zeiss LSM 710 confocal laser scanning microscope (Carl Zeiss Meditec, Jena, Germany). The excitation wavelengths of 6-coumarin and DiI were $488 \mathrm{~nm}$ and $543 \mathrm{~nm}$, respectively.

Furthermore, MDCK cells were seeded in 24-well plates, at a density of $3 \times 10^{5}$ cells/well, and cultured for 3 days. The cells were washed, and PLGA or CS-PLGA nanoparticles containing 6-coumarin were applied to the cell monolayers, at $37^{\circ} \mathrm{C}$. After 2 hours of incubation, the cells were washed five times with ice-cold PBS, detached using trypsin/EDTA, and centrifuged at $1000 \times \mathrm{g}$ for 5 minutes. The amount of nanoparticles taken up by the MDCK cells was measured using a flow cytometer (Moflo ${ }^{\text {TM }}$ XDP High-Speed Cell Sorter; Beckman Coulter Inc, Brea, CA, USA). These treatments were applied to remove the nanoparticles that were potentially adhering to the cell surface.

\section{Investigation of the transport pathway(s) of nanoparticles}

In order to examine the transport pathway utilized by the nanoparticles, the effects of temperature, energy depletion, and endocytic inhibitors on the cellular uptake amount were further studied in the MDCK cells. Cells were seeded at a density of $7.5 \times 10^{4}$ cells/well in Millicell 24-well plates. The transepithelial electrical resistance (TEER) was measured using a Millicell ERS-2 Volt-Ohm meter (EMD
Millipore Corp). The TEER values $\left(\Omega \cdot \mathrm{cm}^{2}\right)$ were calculated according to the following equation:

$$
\mathrm{TEER}=\left(\mathrm{R}-\mathrm{R}_{\text {blank }}\right) \times \mathrm{A}
$$

where $\mathrm{R}$ is the measured resistance across a cell monolayer, $\mathrm{R}_{\text {blank }}$ is the resistance of a blank well, and $\mathrm{A}$ is the surface area of the Millicell filter $\left(0.6 \mathrm{~cm}^{2}\right)$.

\section{Effects of temperature and energy depletion on cellular uptake of nanoparticles}

The MDCK cell monolayers (TEER $\geq 300 \Omega \cdot \mathrm{cm}^{2}$ ) were washed, and $0.5 \mathrm{mg} / \mathrm{mL}$ PLGA or CS-PLGA nanoparticles containing 6-coumarin were applied to cell monolayers, at $37^{\circ} \mathrm{C}$ or $4^{\circ} \mathrm{C}$ for 2 hours. After the incubation, the cells were washed five times with ice-cold PBS and then solubilized with $0.2 \mathrm{~mL}$ of cell lysis reagent E153A (Promega Corp, MadisonFitchburg, WI, USA). The cell lysate solution $(0.2 \mathrm{~mL})$ was added to $3.0 \mathrm{~mL}$ of a mixing solution of methanol/chloroform (1:1), and then, the resultant mixture was centrifuged at $10,000 \times \mathrm{g}$ for 5 minutes. The 6 -coumarin concentrations were determined in the supernatant, with the Fluoroskan Ascent FL microplate reader. Cellular uptake of nanoparticles was expressed as a percentage of the initial amount of nanoparticles.

In addition, the effect of energy depletion on uptake was determined. The total intracellular adenosine $5^{\prime}$-triphosphate (ATP) level was reduced by incubating with sodium azide (10 mM), sodium fluoride (2 $\mathrm{mM})$, and 2-deoxyglucose $(50 \mathrm{mM})$, for 30 minutes at $37^{\circ} \mathrm{C}$. Subsequently, the medium was removed, and nanoparticles containing 6-coumarin were added to the cell monolayers, for 2 hours at $37^{\circ} \mathrm{C}$. The cells were lysed, and the concentration of 6-coumarin was determined with the same method described above.

\section{Effects of endocytic inhibitors on cellular uptake}

Furthermore, various endocytic inhibitors were used to illustrate the involvement of related active transporters in the process of cellular uptake of nanoparticles. The MDCK cell monolayers (TEER $\geq 300 \Omega \cdot \mathrm{cm}^{2}$ ) were washed and preincubated in serum-free DMEM containing amiloride (final concentration, $17.2 \mu \mathrm{g} / \mathrm{mL}$ ) or chlorpromazine (final concentration, $20 \mu \mathrm{g} / \mathrm{mL}$ ) or filipin (final concentration, $1 \mu \mathrm{g} / \mathrm{mL}$ ), for 1 hour, to block macropinocytosis, clathrin-mediated endocytosis, or caveolin-mediated endocytosis, respectively. After the preincubation, the inhibitor solution was replaced with a suspension of nanoparticles $(0.5 \mathrm{mg} / \mathrm{mL})$ containing 6-coumarin, for incubation in the presence of inhibitors, for 
2 hours at $37^{\circ} \mathrm{C}$. The cell monolayers were treated with the method for lysis described above.

\section{Transmembrane permeability studies}

The MDCK cells were seeded in Millicell 24-well cell culture insert plates, at a density of $7.5 \times 10^{4}$ cells/well. The MDCK cell monolayers (TEER $\geq 300 \Omega \cdot \mathrm{cm}^{2}$ ) were washed with HBSS, and then, either a solution of exendin-4, empty nanoparticles mixed with free exendin-4, or exendin-4-loaded nanoparticles (PLGA or CS-PLGA), in which the concentration of exendin- 4 was $5 \mu \mathrm{M}$, was added to the donor (upper) compartment, at $37^{\circ} \mathrm{C}$ for 2 hours. The flux of exendin- 4 in the receiver (lower) compartment was measured with a mass spectrometer (Agilent 1200/6410 LC/MS/MS; Agilent Technologies, Santa Clara, CA, USA) ${ }^{4}$ and the apparent permeability coefficient $\mathrm{P}_{\text {app }}(\mathrm{cm} / \mathrm{s})$ was calculated by the following equation:

$$
\mathrm{P}_{\text {app }}(\mathrm{cm} / \mathrm{s})=(\mathrm{dQ} / \mathrm{dt}) \times\left(1 / \mathrm{A} \times \mathrm{C}_{0}\right)
$$

where $\mathrm{dQ} / \mathrm{dt}$ is the increase in the amount of drug in the receiver chamber per time interval, $\mathrm{C}_{0}$ is the initial concentration in the donor compartment, and $\mathrm{A}$ is the permeation area of the cell culture insert.

\section{In vivo evaluation of exendin-4-loaded nanoparticles in rats}

Wistar male rats, weighing 200-230 g, were obtained from the Experimental Animal Center of the Norman Bethune College of Medicine (Jilin University, Changchun, People's Republic of China). The rats were cared for in accordance with the guidelines of the Animal Use Committee of Jilin University for the use and care of laboratory animals. The rats were housed in groups of 6-8, under a 12-hour light/dark cycle, and were allowed food and water ad libitum. The rats were fasted overnight (approximately 18 hours) before the experiment but had free access to water. After each rat was anesthetized with $1 \%$ pentobarbital sodium $(4.0 \mathrm{~mL} / \mathrm{kg}$ by intraperitoneal injection), a midline laparotomy was performed to expose the abdomen. The first $10 \mathrm{~cm}$ of intestine (duodenum) was prepared by washing three times with physiological saline. The rats were divided into four control groups and two treatment groups. The control groups 1, 2, 3, and 4 were administered saline, empty CSPLGA nanoparticles in saline solution, a saline solution of free exendin-4 $(500 \mu \mathrm{g} / \mathrm{kg})$, and empty CS-PLGA nanoparticles mixed with free exendin-4 solution $(500 \mu \mathrm{g} / \mathrm{kg})$, respectively. Groups 5 and 6 were administered exendin-4-loaded PLGA nanoparticles and exendin-4-loaded CS-PLGA nanoparticles, respectively, in which the concentrations of exendin-4 were both at $500 \mu \mathrm{g} / \mathrm{kg}$. After the administration, both ends of the duodenum were ligated. Blood samples were collected from the heart at specified time intervals $(15,30,45,60$, 90,120 , and 180 minutes) and were centrifuged $(1,600 \times \mathrm{g}$, 20 minutes). The concentration of exendin- 4 was determined by using an extendin-4 immunoassay (EIA) kit (EK-070-94; Phoenix Pharmaceuticals Inc, Burlingame, CA, US).

\section{Statistical analysis}

All results are expressed as the mean \pm standard deviation (SD) of more than three replicates. Statistical comparisons were performed using the Student's $t$-test for two groups, with a $P$-value of less than 0.05 being considered statistically significant.

\section{Results \\ Physicochemical properties of nanoparticles}

Physicochemical properties of the PLGA and CS-PLGA nanoparticles were characterized in terms of size and zeta potential, and the results are summarized in Table 1. The mean particle size of the PLGA nanoparticles was $274.6 \pm 4.1 \mathrm{~nm}$, smaller than that of the CS-PLGA nanoparticles (around $300 \mathrm{~nm}$ ). The zeta potential of the PLGA nanoparticles was $-13.8 \pm 2.9 \mathrm{mV}$, whereas the CS-PLGA nanoparticles were positively charged, with a higher zeta potential of $+17.0-24.7 \mathrm{mV}$. These results suggested that the PLGA nanoparticles were successfully coated with chitosan.

For chitosan of the same molecular weight, the zeta potential of CS-PLGA nanoparticles steadily rose with the increasing degree of deacetylation of chitosan, whereas there was no significant change observed in particle size. At the same degree of deacetylation, the zeta potential of the CSPLGA nanoparticles was elevated along with the increase in molecular weight of chitosan; however, the effect on particle size was not apparent. Therefore, both the molecular weight and degree of deacetylation of chitosan showed a significant influence on the zeta potential of the CS-PLGA nanoparticles but not on the particle size, under our experimental conditions. The effect of the concentration of chitosan (400 kDa, 95\% degree of deacetylation) on the characteristics of CS-PLGA nanoparticles was also investigated. As shown in Figure 1A, the particle size increased from 287 to $337 \mathrm{~nm}$, with increasing concentrations of chitosan; however, the zeta potential remained unchanged except for the first concentration $(0.3 \mathrm{mg} / \mathrm{mL})$, which was slightly lower than the others (Figure 1B). 
Table I Particle size and zeta potential of PLGA and CS-PLGA nanoparticles (mean \pm SD, $n=3$ )

\begin{tabular}{|c|c|c|c|c|c|c|c|}
\hline & \multirow[t]{2}{*}{ PLGA NPs } & \multicolumn{6}{|c|}{ CS-PLGA NPs } \\
\hline & & $85 \%$ a, $200^{b}$ & $85 \%^{\mathrm{a}}, 300^{\mathrm{b}}$ & $85 \%^{\mathrm{a}}, 400^{\mathrm{b}}$ & $95 \%$ a, $200^{b}$ & $95 \%$ a, $300^{b}$ & $95 \%^{\mathrm{a}}, 400^{\mathrm{b}}$ \\
\hline Particle size $(\mathrm{nm} \pm \mathrm{SD})$ & $274.6 \pm 4.1$ & $30 I .7 \pm 3.6$ & $300.4 \pm 1.4$ & $295.9 \pm 4.6$ & $292.4 \pm 3.8$ & $309.8 \pm 2.6$ & $291.5 \pm 3.1$ \\
\hline Zeta potential $(m \mathrm{~m} \pm \mathrm{SD})$ & $-13.8 \pm 2.9$ & $17.0 \pm 2.5$ & $18.8 \pm 1.7$ & $20.0 \pm 1.9$ & $20.4 \pm 2.0$ & $22.9 \pm 0.7$ & $24.7 \pm 1.1$ \\
\hline
\end{tabular}

Notes: ${ }^{a}$ The degree of deacetylation of chitosan, expressed as a percentage; b the chitosan molecular weight, expressed in kDa.

Abbreviations: CS-PLGA NPs, chitosan-coated poly (d,I-lactide-co-glycolide) nanoparticles; PLGA NPs, poly (d,I-lactide-co-glycolide) nanoparticles; SD, standard deviation.

\section{Cytotoxicity of nanoparticles}

The potential cytotoxicity of the nanoparticles was evaluated by MTT assay after a 2-hour incubation with MDCK cells (Figure 2). Both the PLGA nanoparticles and CS-PLGA nanoparticles showed dose-dependent negative effects on MDCK cell viability, with the latter resulting in a slightly lower level of cell viability. However, even at the maximum concentration of $5 \mathrm{mg} / \mathrm{mL}$ used in this study, cell viabilities of more than $80 \%$ were obtained with all tested samples of CS-PLGA nanoparticles, suggesting an acceptable level of biocompatibility.
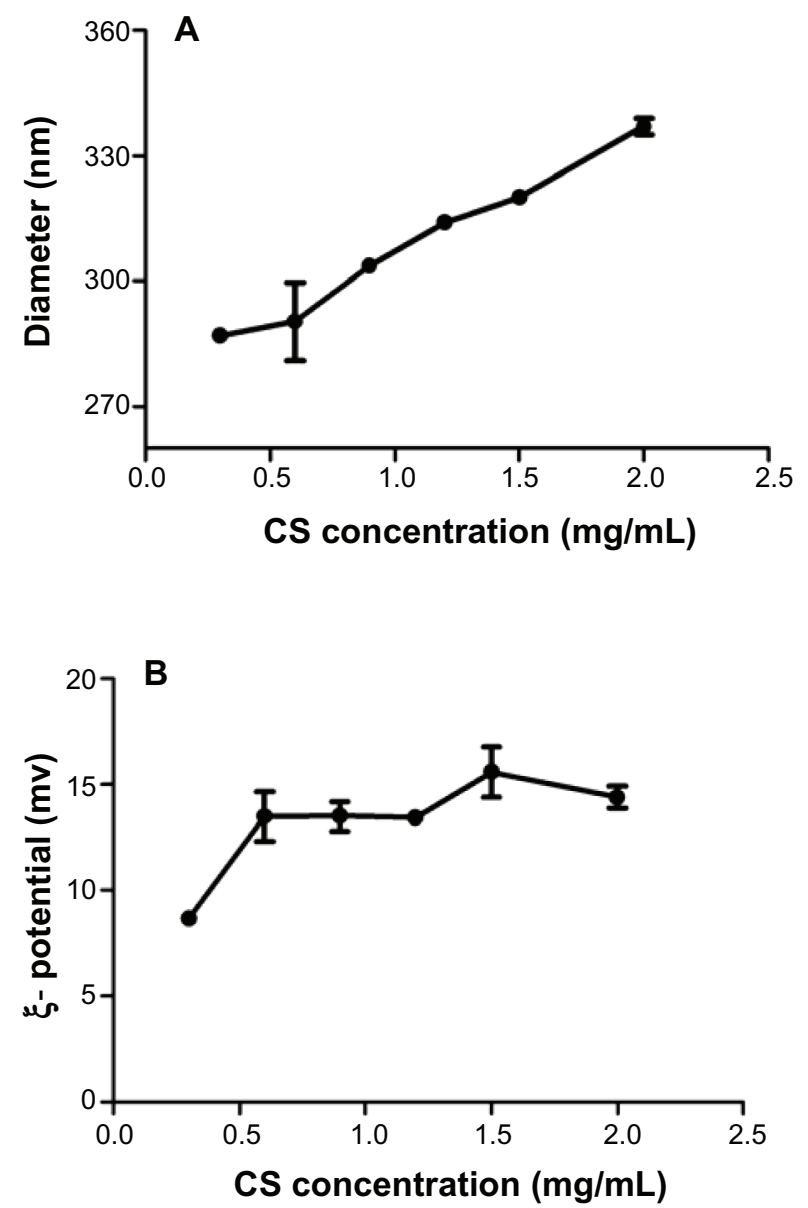

Figure I Effects of chitosan concentrations on (A) particle size and (B) zeta potential of nanoparticles.

Note: Data represent means \pm SD $(n=3)$.

Abbreviations: $\mathrm{CS}$, chitosan; SD, standard deviation.

\section{Interaction of nanoparticles with MDCK cells}

In the subsequent studies, 6-coumarin, as a fluorescent probe, was loaded into nanoparticles of the same particle size and zeta potential as those of the exendin-4-loaded nanoparticles to examine the interaction of the nanoparticles with the MDCK cells. First, the effect of the concentration of nanoparticles on cellular uptake was investigated. As seen in Figure 3A, the uptake of both PLGA and CS-PLGA nanoparticles into cells increased linearly as a function of concentration in the range of $0-0.8 \mathrm{mg} / \mathrm{mL}$ and thereafter reached a plateau phase. The effect of incubation time on the cellular uptake was also investigated in the 96-well cell plates (Figure 3B). The results showed that the fluorescence intensity sharply increased during the first 30 minutes of incubation, followed by a slight change in the rate of uptake into the MDCK cell monolayer, and saturation was reached by 2 hours. In addition, at all concentrations and time points, the amount of CS-PLGA nanoparticles taken up by the cells (including solid surface adsorption and internalization) was significantly higher than that of PLGA. Moreover, it is worth noting that there was no detectable fluorescence signal from

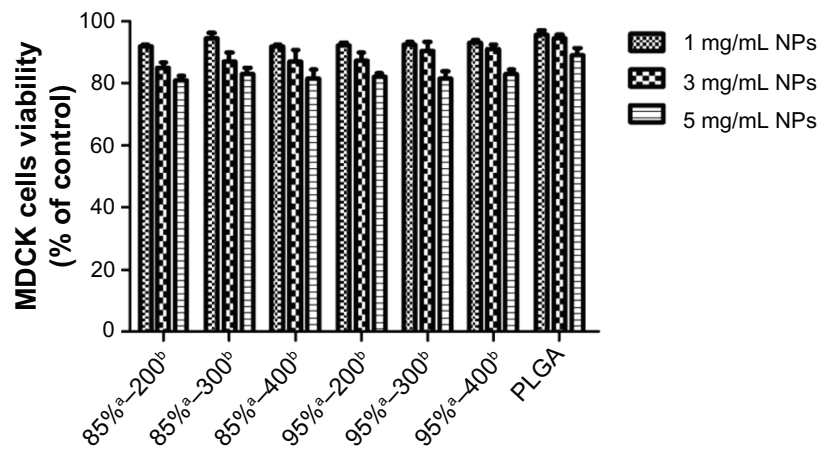

Figure 2 Effects of nanoparticles on the viability of MDCK cells. Notes: MDCK cells were incubated with PLGA nanoparticles or PLGA nanoparticles coated with chitosans of variable molecular weights and degrees of deacetylation, for 120 minutes and then processed for the MTT assay. Results are expressed as a percentage of the initial value and presented as means \pm SD $(n=5)$. ${ }^{\text {Represents }}$ the degree of deacetylation of chitosan, expressed as a percentage; 'bepresents the chitosan molecular weight, expressed in $\mathrm{kDa}$.

Abbreviations: MDCK, Madin-Darby canine kidney; MTT, 3-(4,5-dimethylthiazol2-yl)-2,5-diphenyltetrazolium bromide; PLGA, poly (d,I-lactide-co-glycolide); $\mathrm{SD}$, standard deviation. 

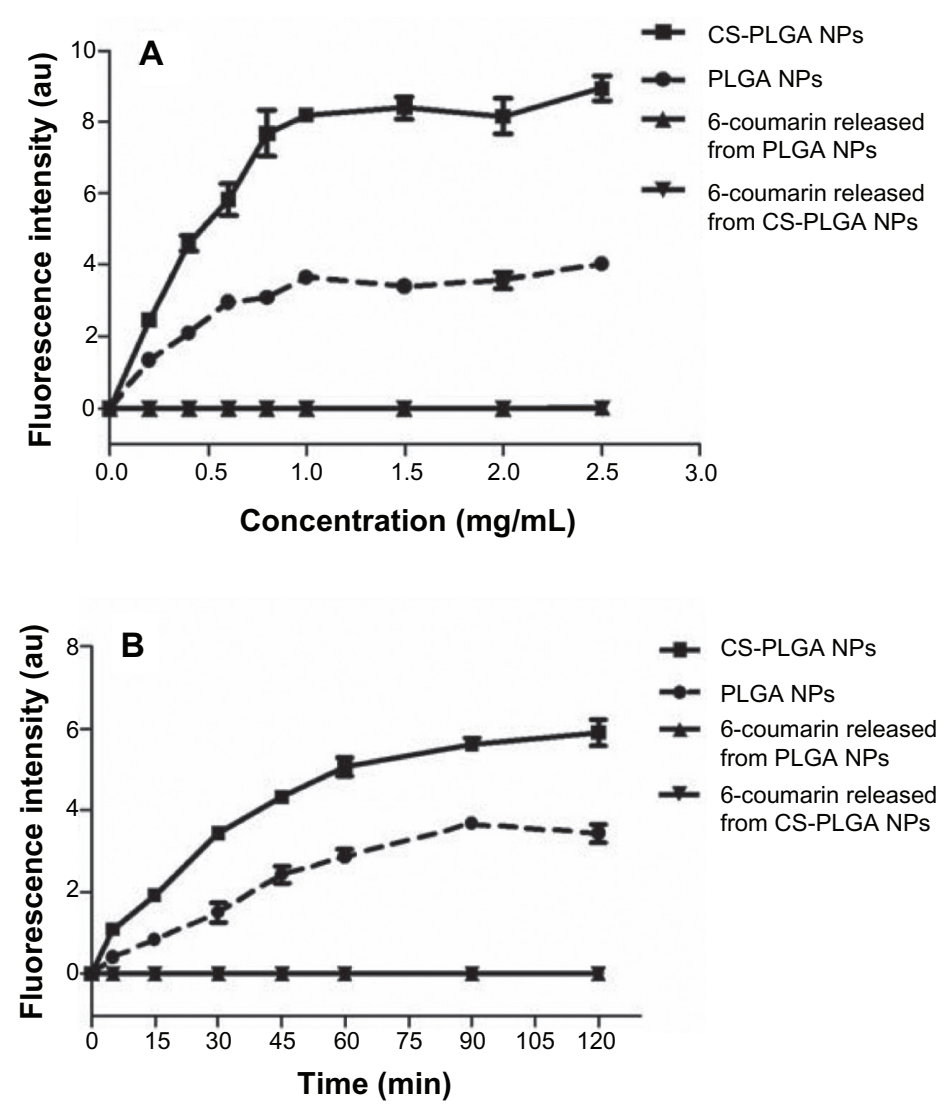

Figure 3 Effects of (A) concentration and (B) incubation time, on the cellular uptake of nanoparticles and free 6-coumarin released from PLGA or CS-PLGA nanoparticles.

Notes: MDCK cells were seeded in 96-well plates and incubated with PLGA, or CS-PLGA nanoparticles, or equivalent amount of free 6-coumarin released from PLGA or CS-PLGA nanoparticles, at $37^{\circ} \mathrm{C}$. The fluorescence intensity of the internalized nanoparticles or free 6-coumarin was detected using a Fluoroskan Ascent FL microplate reader. Data are means \pm SD, of five different cell monolayers.

Abbreviations: CS-PLGA, chitosan-coated poly (d,I-lactide-co-glycolide); NP, nanoparticle; MDCK, Madin-Darby canine kidney; PLGA, poly (d,I-lactide-co-glycolide); $S D$, standard deviation.

the cells incubated with the equivalent concentrations of free 6-coumarin released from the PLGA and CS-PLGA nanoparticles, respectively, which indicated that the fluorescence determined in abovementioned experiments resulted from the 6-coumarin loaded into the nanoparticles. The cellular uptake of 6-coumarin-loaded nanoparticles was visually observed using confocal laser scanning microscopy (Figure 4A), and the cellular membrane of the MDCK monolayer was noted by the localization of DiI (red). Figure 4B and C show the green fluorescent 6-coumarin-loaded nanoparticles in the cytoplasm, suggesting that both PLGA and CS-PLGA nanoparticles were internalized by the MDCK cells. The yellow color in the images indicates the colocalization of 6-coumarin-loaded nanoparticles (green) and the cellular membrane (red). Although a few particles were also found on the cell surface, most of the PLGA nanoparticles were present inside the cells. In order to rule out the possible contribution of adhesion of nanoparticles to the cellular surface, the uptake of nanoparticles was further evaluated by the flow cytometric analysis of cells that were treated with trypsin and extensively washed after the coincubation, to remove plasma membrane-bound nanoparticles (Figure 5). The results showed that the fluorescence intensity obtained with the CS-PLGA nanoparticles was significantly higher than that measured after exposure to the PLGA nanoparticles $(P<0.05)$, indicating the higher cellular transport potential of CS-PLGA nanoparticles.

In order to identify the uptake mechanism of these nanoparticles, the effect of temperature on cellular uptake was investigated by measuring the fluorescence of 6-coumarin in the cells after 2 hours of coincubation, at $37^{\circ} \mathrm{C}$ or $4^{\circ} \mathrm{C}$. The concentration of $0.5 \mathrm{mg} / \mathrm{mL}$ of nanoparticles, which was in the linear range, was used in subsequent experiments. As shown in Figure 6A, cellular uptake levels of the PLGA and CS-PLGA nanoparticles at $4^{\circ} \mathrm{C}$ were significantly decreased, by about eightfold and fourfold, respectively, in comparison with those at $37^{\circ} \mathrm{C}$. In addition, cellular uptake levels of the PLGA and CS-PLGA nanoparticles in the ATP-depleted 

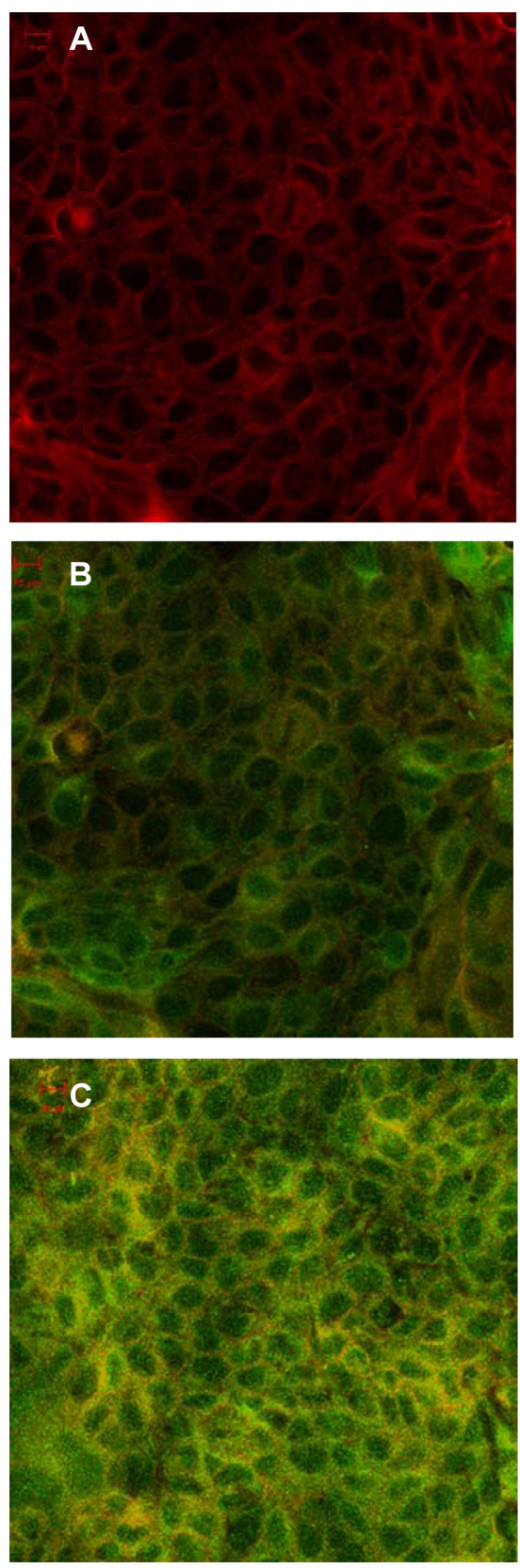

Figure 4 Confocal laser microscopy images of MDCK cells incubated for $2 \mathrm{~h}$ at $37^{\circ} \mathrm{C}$ (A) without or (B) with a suspension of 6-coumarin-(green) loaded $(0.2 \mathrm{mg} / \mathrm{mL})$ PLGA nanoparticles or (C) CS-PLGA nanoparticles.

Notes: For all samples, MDCK cellular membranes were stained with Dil (red), and the appearance of yellow color indicates colocalization of nanoparticles with the cellular membrane. Scale bar, $10 \mu \mathrm{m}$.

Abbreviations: CS-PLGA, chitosan-coated poly (d,l-lactide-co-glycolide); Dil, I,I'-dioctadecyl-3,3,3',3'-tetramethylindocarbocyanine perchlorate; MDCK, Madin-Darby canine kidney; PLGA, poly (d,I-lactide-co-glycolide).

cells treated with sodium azide, sodium fluoride, and 2-deoxyglucose were reduced by fourfold and twofold, respectively (Figure 6B). These results showed that the uptake of PLGA and CS-PLGA nanoparticles by the MDCK cell monolayer involved active transport. Furthermore, inhibition of caveolin-mediated endocytosis by filipin decreased the intracellular uptake of PLGA nanoparticles by $17 \%$, whereas inhibition of endocytosis by chlorpromazine and amiloride did not affect the uptake of these nanoparticles, indicating that neither clathrin nor macropinocytosis were involved (Figure 6C). In the case of CS-PLGA nanoparticles, their uptake was not affected by filipin, suggesting that caveolin-mediated endocytosis did not play an important role; however, inhibition by chlorpromazine or by amiloride resulted in a similar increase, of $23 \%$, in cellular uptake, indicating that clathrin-mediated endocytosis and macropinocytosis were both involved in the intracellular uptake of these nanoparticles.

\section{Transmembrane permeability enhancement}

To evaluate the potential enhancement effect of chitosan coating on the transmembrane permeability of the nanoparticles, the $\mathrm{P}_{\text {app }}$ value of exendin-4 in various formulations was determined in the MDCK cell model after 2 hours of coincubation at $37^{\circ} \mathrm{C}$. As shown in Figure 7, the $\mathrm{P}_{\text {app }}$ of the exendin- 4 solution was around $0.17 \times 10^{-6}$, and the presence of either empty PLGA or CS-PLGA nanoparticles did not affect the transport of free exendin- 4 across the MDCK cell monolayers. The encapsulation of exendin-4 into the PLGA nanoparticle resulted in an increase in transmembrane permeability up to $1.52 \times 10^{-6}$, whereas the exendin-4-loaded PLGA nanoparticles coated with the various chitosans displayed $\mathrm{P}_{\text {app }}$ values in the range of 2.5-3.0 $\times 10^{-6}$. The results indicated that both types of nanoparticles were able to increase exendin- 4 transmembrane permeability by 8.9 to 16.5 -fold, with CS-PLGA nanoparticles having a greater effect. Interestingly, the coating of PLGA with chitosan at variable degrees of deacetylation did not have a significant effect on the $\mathrm{P}_{\text {app }}$ of exendin- 4 .

\section{In vivo evaluation of exendin-4-loaded nanoparticles in rats}

To further evaluate the effectiveness of CS-PLGA nanoparticles in oral delivery of exendin-4, intraduodenal administration was performed in rats. The plasma exendin- 4 concentrations of various formulations at various time points are shown in Figure 8. As expected, at all time points, exendin- 4 could not be detected in the groups administered saline or empty CS-PLGA nanoparticles, which were used as negative controls. In addition, there was no detectable exendin- 4 in the groups administered exendin-4 saline solution or empty CS-PLGA nanoparticles mixed with exendin-4, whereas both exendin-4-loaded nanoparticles showed high plasma concentrations, of $0.30 \pm 0.15 \mathrm{ng} / \mathrm{mL}$ and $0.50 \pm 0.17 \mathrm{ng} / \mathrm{mL}$ for the PLGA and CS-PLGA nanoparticles, respectively, at 2 hours after administration. At 1.5, 2, and 

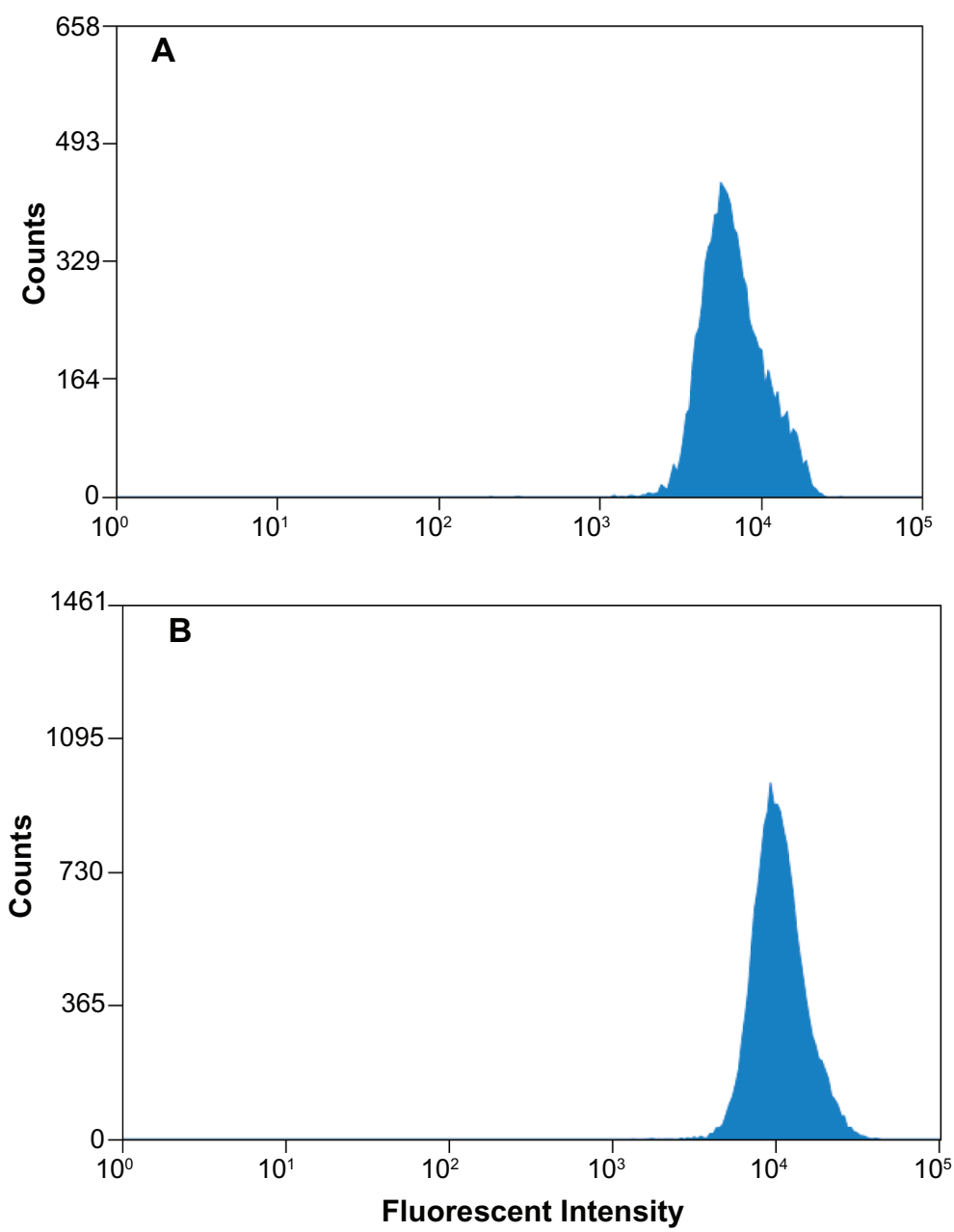

Figure 5 Quantitative evaluation of the interactions of nanoparticles with MDCK cells. The images show histogram profiles of fluorescence intensities in cells following exposure to equivalent amounts of (A) 6-coumarin-loaded PLGA and (B) 6-coumarin-loaded CS-PLGA nanoparticles, after $2 \mathrm{~h}$ of incubation at $37^{\circ} \mathrm{C}$. Abbreviations: CS-PLGA, chitosan-coated poly (d,I-lactide-co-glycolide); MDCK, Madin-Darby canine kidney; PLGA, poly (d,I-lactide-co-glycolide).

3 hours, the plasma drug levels of the group receiving CSPLGA nanoparticles were much higher than those of the group receiving PLGA nanoparticles, and significant differences were observed at 1.5 and 3 hours $(P<0.05)$.

\section{Discussion}

\section{Characteristics of surface-modified PLGA nanoparticles}

The particle size and zeta potential of nanoparticles are critical parameters for their biological application. Tahara et $\mathrm{al}^{16}$ studied the effect of different particle sizes (200, 400, and $1000 \mathrm{~nm}$ ) on the interaction of nanoparticles with A549 cells and found that the cellular uptake of nanoparticles increased when the size was controlled below $400 \mathrm{~nm}$. Another study also revealed that the interaction of nanoparticles with cells correlated with the particle size, with smaller particles generally resulting in higher cellular uptake. ${ }^{24}$ However, the drug-loading efficiency of nanoparticles will decrease with smaller particles. Therefore, it is essential to balance the particle size and drug-loading efficiency, for practical applications. In the present study, $300 \mathrm{~nm}$ was chosen as the upper limit of nanoparticle size. In addition, compared with the unmodified PLGA nanoparticles, all CS-PLGA nanoparticles displayed a slightly larger size, and their zeta potential was significantly changed from negative to positive, suggesting the successful coating of PLGA nanoparticles with all concentrations of chitosan.

It has been reported that chitosan can adhere to the surface of negatively charged PLGA nanoparticles by electrostatic attraction to form the first monomolecular adsorption layer. Thereafter, the hydrogen bond or van der Waal's force would be the dominant driving force for the further adsorption of chitosan. ${ }^{23,25}$ During the formation of the first few molecular adsorption layers, the chitosan density on the surface of PLGA nanoparticles increases with increasing concentrations of chitosan. ${ }^{25}$ As a result, 

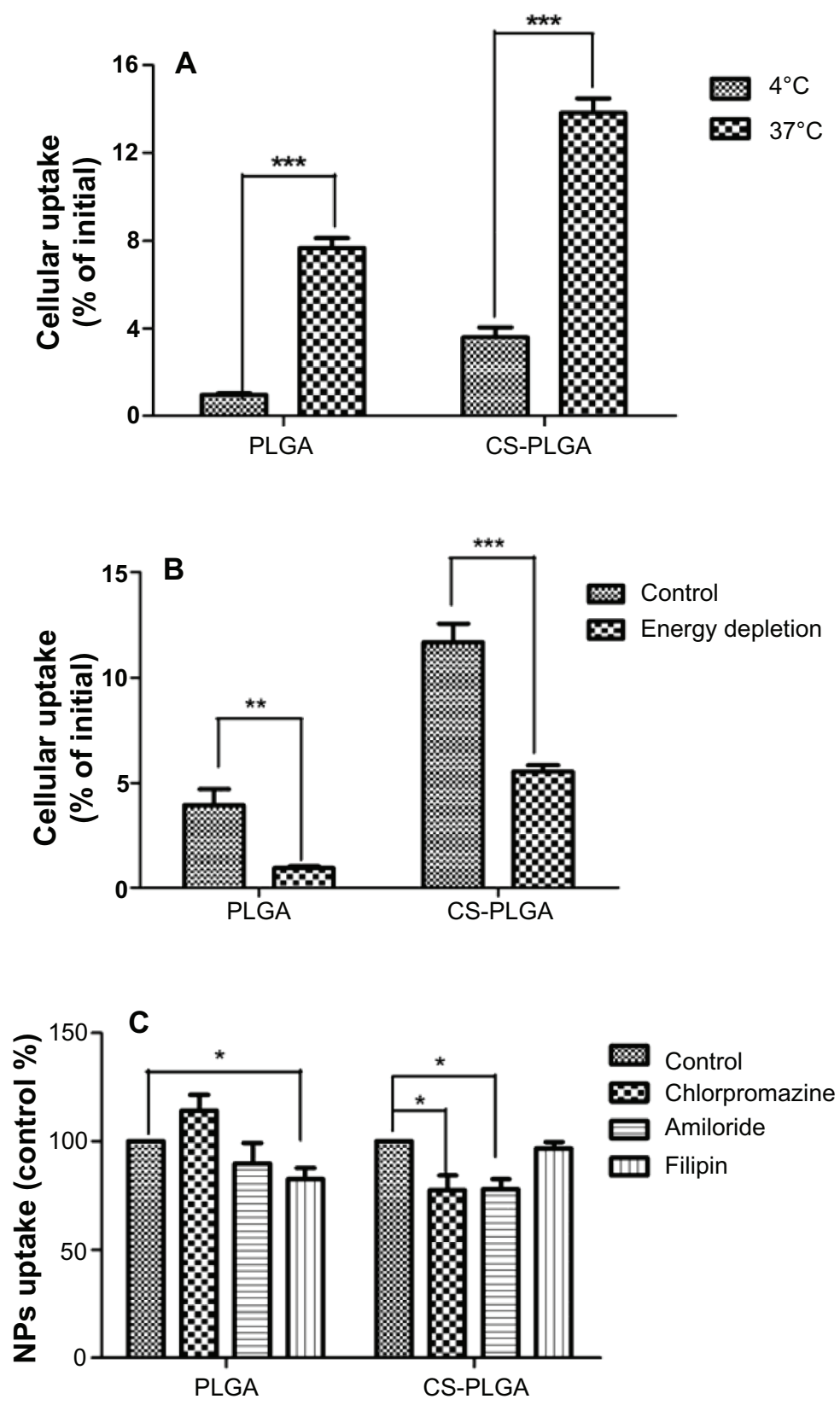

Figure 6 Effects of temperature, energy depletion, and endocytic inhibitors on cellular uptake of nanoparticles. MDCK cell monolayers were seeded in 24-well cell culture insert plates and incubated with PLGA or CS-PLGA nanoparticles $(\mathbf{A})$ at different temperatures $\left(4^{\circ} \mathrm{C}\right.$ and $\left.37^{\circ} \mathrm{C}\right)$; (B) without or with depletion of energy; and $(\mathbf{C})$ in the presence of the clathrin-mediated endocytosis inhibitor chlorpromazine $(20 \mu \mathrm{g} / \mathrm{mL})$, the caveolin-mediated endocytosis inhibitor filipin (I $\mu \mathrm{g} / \mathrm{mL})$, or the macropinocytosismediated endocytosis inhibitor amiloride $(17.2 \mu \mathrm{g} / \mathrm{mL})$.

Notes: Data are means $\pm S D$, of five different cell monolayers. $* P<0.05 ; * * P<0.01 ; * * * P<0.001$

Abbreviations: CS-PLGA, chitosan-coated poly (d,I-lactide-co-glycolide); MDCK, Madin-Darby canine kidney; NP, nanoparticle; PLGA, poly (d,I-lactide-co-glycolide); SD, standard deviation.

the zeta potential of the nanoparticles increases, without a significant change in particle size. However, layers beyond the first few will not increase the zeta potential, due to the constant apparent surface charge per unit area (amine groups), but will result in a significant increase in particle size. These stepwise coating processes of chitosan can reasonably explain the result obtained in the present study. When the coating concentration of chitosan increased from
0.3 to $0.6 \mathrm{mg} / \mathrm{mL}$, the zeta potential showed a significant increase, accompanied by a slight change in particle size (Figure 1). At concentrations higher than $0.6 \mathrm{mg} / \mathrm{mL}$, the zeta potential reached a plateau, accompanied by a significant increase in particle size, suggesting that the first few molecular absorption layers formed at the concentration of $0.6 \mathrm{mg} / \mathrm{mL}$. Interestingly, no apparent change in particle size was observed in the presence of chitosan with variable 


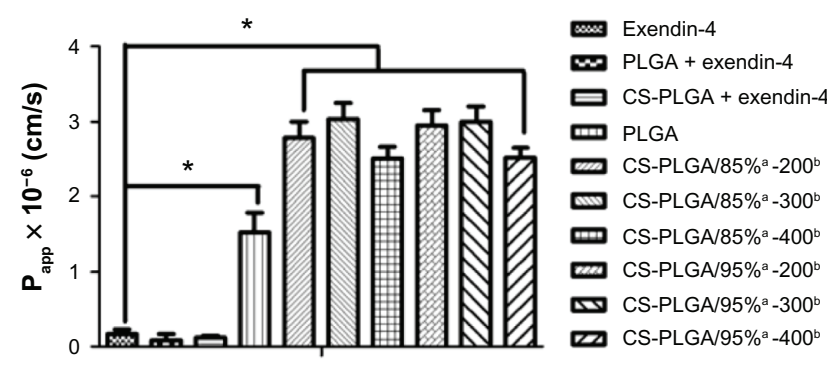

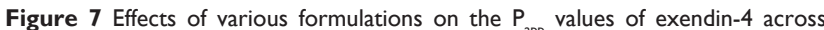
MDCK cell monolayers, after $2 \mathrm{~h}$ of incubation at $37^{\circ} \mathrm{C}$.

Notes: The concentration of exendin- 4 was $5 \mu \mathrm{M}$ in all groups. Data are means $\pm S D$,

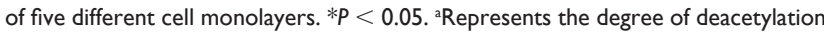
of chitosan, expressed as a percentage; 'bepresents the chitosan molecular weight, expressed in $\mathrm{kDa}$.

Abbreviations: CS-PLGA, chitosan-coated poly (d,I-lactide-co-glycolide); MDCK, Madin-Darby canine kidney; $P_{\text {app }}$, apparent permeability coefficient; PLGA, poly (d,Ilactide-co-glycolide); SD, standard deviation.

molecular weights at the same concentration of $0.6 \mathrm{mg} / \mathrm{mL}$ (Table 1), which was probably due to the limited conformation of the chitosan chains absorbed onto the surface of the PLGA nanoparticles. ${ }^{25}$ In addition, the results of the MTT toxicity assay demonstrated that the nanoparticles coated with chitosan of variable molecular weights and degrees of deacetylation had only slight cytotoxicity, as they reduced the MDCK cell growth by less than 20\% (Figure 2).

\section{Cellular uptake and interaction between nanoparticles and cells}

MDCK cells, derived from a normal male cocker spaniel, can differentiate into columnar epithelium and form tight

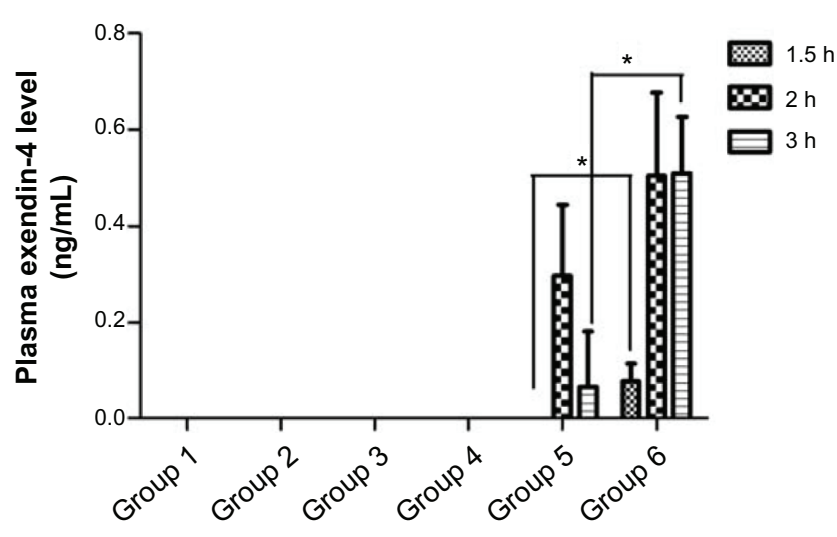

Figure 8 Plasma exendin-4 levels over time, following the administration of different formulations.

Notes: Control groups I, 2, 3, and 4 were administered saline, empty CS-PLGA nanoparticles in saline solution, a saline solution of free exendin-4 $(500 \mu \mathrm{g} / \mathrm{kg})$, and empty CS-PLGA nanoparticles mixed with free exendin-4 solution $(500 \mu \mathrm{g} / \mathrm{kg})$ respectively. Groups 5 and 6 were administered exendin-4 $(500 \mu \mathrm{g} / \mathrm{kg})$ loaded PLGA and CS-PLGA nanoparticles, respectively. Data are means \pm SD $(n=3)$. $* P<0.05$

Abbreviations: CS-PLGA, chitosan-coated poly (d,I-lactide-co-glycolide); PLGA, poly (d,I-lactide-co-glycolide); SD, standard deviation. junctions similar in physiology to that of the small intestine; therefore, these have been widely used to study the transport of drugs and artificial carriers. ${ }^{26-28}$ In this study, the MDCK cell monolayer model was chosen to investigate the transport characteristics and related mechanism of PLGA and CS-PLGA nanoparticles. The highly sensitive fluorescent probe 6-coumarin was encapsulated in particles to analyze their cellular uptake. It should be noted that the percentages of 6-coumarin dye released from the PLGA and CS-PLGA nanoparticles were only between $0.06 \%-0.38 \%$ during a 24-hour period, indicating that the 6-coumarin detected in the cells was mainly associated with the nanoparticles. This hypothesis is well supported by the data obtained from the experiments performed by using equivalent amounts of the free 6-coumarin released from the PLGA and CS-PLGA nanoparticles (Figure 3A and B). Another study has also shown that 6-coumarin alone cannot be directly internalized by the cells. ${ }^{29}$

The transport pathways in epithelial cells include paracellular transport, passive transcellular diffusion, and active transporter-mediated transcellular and transcytosis transport. Generally, transporter-mediated active transport is saturable and energy-dependent. The cellular uptake of both nanoparticle formulations exhibited a saturated pattern with increasing concentrations and incubation times, which suggested the involvement of active transport. This speculation was supported in subsequent experiments evaluating the effects of temperature and energy depletion on uptake of the nanoparticles, and the results were similar to those described using A549 cells. ${ }^{16}$ Further investigation revealed that the active transport of the PLGA nanoparticles into cells involved caveolin-mediated endocytosis. However, the chitosan coating changed the transport mechanism of the PLGA nanoparticles, for which the uptake was inhibited by both chlorpromazine and amiloride, suggesting the involvement of clathrin-mediated endocytosis and macropinocytosis. The different modes of cellular uptake of the PLGA and CS-PLGA nanoparticles into the MDCK cells may be due to their different properties, such as particle size and surface charge, which would therefore result in different interactions with the cell membrane and transporters. Clathrin-mediated endocytosis has been reported to mediate uptake of small particles, with the upper limit of about $200 \mathrm{~nm}$; meanwhile, the transport mechanism switched to caveolae-mediated internalization for particles of increased size, and no uptake was seen for $1 \mu \mathrm{m}$ particles..$^{30}$ On the other hand, the uptake of octa arginine-modified liposomes with positive charges has been found to be inhibited by treatment with chlorpromazine 
and amiloride in the MDCK cell model, indicating that the modified liposomes are internalized via clathrin-mediated endocytosis and macropinocytosis. In contrast, cellular uptake of unmodified liposomes is not prevented by any of these inhibitors, and their internalization may occur by an unknown pathway. ${ }^{31}$ Tahara et al $^{16}$ has reported that both PLGA and CS-PLGA nanoparticles are taken up in A549 cells through clathrin-mediated endocytosis, but the contrasting results with the findings of our study may be due to the different cell models.

Besides the different transport mechanisms, it is worth noting that, under all experimental conditions, higher cellular uptake was observed with the chitosan-modified PLGA nanoparticles, which may be attributed to their positively charged surface. The greater advantage of modified over unmodified PLGA nanoparticles in cellular uptake was also evidenced by confocal microscopic observations. In order to eliminate nanoparticles potentially absorbed to cell surfaces, trypsin was used to treat the cells after the incubation and before the flow cytometric analysis. As expected, higher fluorescence intensities were observed in the MDCK cells incubated with CS-PLGA particles, compared with those incubated with PLGA particles $(P<0.05)$. The greater advantage of CS-PLGA nanoparticles in cellular uptake over PLGA nanoparticles may be attributed to the electrostatic interactions between the positively charged amino acid groups of chitosan and the negatively charged cell membrane, thereby altering the subsequent endocytosis process through different pathways. ${ }^{16,21,24}$

\section{Transmembrane permeability and in vivo delivery of exendin- 4 in rats}

In our previous work, the transport of free exendin- 4 across the MDCK cell monolayer was determined to be very limited and to occur primarily via paracellular passive diffusion. ${ }^{4}$ Extendin-4 is a Class III drug, with high solubility and low permeability, in the Biopharmaceutics Classification System. To improve the cellular uptake of exendin-4, CS-PLGA nanoparticles were used as the transport vector in the present study. The above results revealed that both exendin-4-loaded PLGA and CS-PLGA nanoparticles could be significantly taken into cells via an active transport mechanism. However, efficient cellular uptake certainly does not guarantee significant transport across the cell monolayer. The previously reported failure of chitosan nanoparticle-mediated insulin transport across the Caco-2 cell monolayer was explained by the inability of nanoparticles to breach the basal membrane of cells. ${ }^{32}$ Generally, efficient cellular uptake followed by the retention of carriers is advantageous for the intracellular delivery of therapeutic genes and drugs. ${ }^{33}$ However, good transmembrane permeability is a fundamental requirement for efficient oral absorption and systemic availability.

It has been reported that the PLGA nanoparticles are entrapped in the endosomes after internalization into cells, from where they are sorted to late endosomes and lysosomes or to the trans-Golgi network. ${ }^{34,35}$ The fraction of PLGA nanoparticles entrapped in lysosomes may suffer from degradation, whereas the others transported to the transGolgi network can be packaged into secretory vesicles for exocytosis through fusion with the basolateral membrane. ${ }^{36}$ Panyam et $\mathrm{l}^{37}$ suggested a rapid endolysosomal escape into the cytosol by PLGA nanoparticles, due to their selective reversal of the surface charge (from anionic to cationic) in the acidic endolysosomal compartment. The similar phenomenon of endolysosomal escape was also reported for chitosanmodified iron oxide nanoparticles, ${ }^{38}$ which probably results from the "proton-sponge" effect.

In this study, encapsulation of exendin-4 into PLGA and CS-PLGA nanoparticles was found to significantly improve the $\mathrm{P}_{\text {app }}$ value of exendin-4, by 8.9- to 16.5-fold, respectively, compared with that of exendin-4 alone $(P<0.05)$. The CSPLGA nanoparticles also showed a higher potential for cellular uptake, with a $P_{\text {app }}$ value of $2.5-3.0 \times 10^{-6} \mathrm{~cm} / \mathrm{s}$, which was likely attributed to interactions between the positively charged particle surface and negatively charged cell membrane. ${ }^{26}$ The improved $\mathrm{P}_{\text {app }}$ value may have resulted from the exocytosis of nanoparticles following endolysosomal escape or the exocytosis of exendin-4 released from nanoparticles into cytoplasm. ${ }^{36,37}$ Further investigation is needed to illustrate the detailed mechanism.

In addition, no significant difference was observed between nanoparticles modified with various chitosans under our experimental conditions, probably due to their similar physicochemical characteristics. The exception of the slight decrease in permeability with increasing molecular weight may be explained by the higher zeta potentials and therefore, the stronger interactions with cells. Moreover, the in vivo evaluation also showed that both PLGA and CS-PLGA nanoparticles could improve the adsorption of exendin-4 in the duodenum, whereas no detectable amount was found with free exendin-4 or empty nanoparticles (both PLGA and CS-PLGA) mixed with free exendin-4. In contrast, the administration of CS-PLGA nanoparticles to rats resulted in higher plasma drug levels and longer retention times than those with PLGA nanoparticles. The results of the in vivo experiment were also consistent with the results of the transmembrane permeability analysis. Collectively, the 
findings of this study suggest that chitosan-modified PLGA nanoparticles can be used as a potential delivery carrier, to improve the bioavailability of exendin- 4 .

\section{Conclusion}

In this study, CS-PLGA nanoparticles were prepared with chitosan of various molecular weights and degrees of deacetylation, and their potential use for delivery of exendin- 4 and related mechanisms also were investigated in detail. The chitosan coating at the concentration of $0.6 \mathrm{mg} / \mathrm{mL}$ led to a significant change in zeta potential, from negative to positive, accompanied by an increase in particle size of $\sim 30 \mathrm{~nm}$, suggesting the formation of multiple molecular adsorption layers around the PLGA nanoparticles. The coating with chitosan also changed the cellular-uptake mechanisms of the nanoparticles, as evidenced by the use of endocytic inhibitors. The uptake of the PLGA nanoparticles occurred via caveolin-mediated endocytosis, whereas the uptake of the CS-PLGA nanoparticles involved both macropinocytosis and clathrin-mediated endocytosis. However, under all conditions, the CS-PLGA nanoparticles showed a higher potential to be transported into cells, as shown by flow cytometry and confocal microscopy. Transmembrane permeability analysis showed that unmodified and modified PLGA nanoparticles could improve the transport of exendin-4 by up to 8.9- and 16.5-fold, respectively, consistent with the evaluation in rats. This study demonstrates that chitosan-coated nanoparticles have a higher transport potential over both free drug and unmodified nanoparticles, providing support for their potential development as a candidate oral delivery agent for exendin-4. Further investigations are required to identify their hypoglycemic efficacy in type 2 mutant diabetic $(\mathrm{db} / \mathrm{db})$ mice.

\section{Acknowledgments}

This work was supported by the National Natural Science Foundation of China (No 30901863 and No 81201764) and the Fundamental Research Funds for the Central Universities (201103184).

\section{Disclosure}

The authors report no conflicts of interest in this work.

\section{References}

1. Li X, Li L, Wang X, Ren Y, Zhou T, Lu W. Application of model-based methods to characterize exenatide-loaded double-walled microspheres: in vivo release, pharmacokinetic/pharmacodynamic model, and in vitro and in vivo correlation. J Pharm Sci. 2012;101(10):3946-3961.

2. Mahato RI, Narang AS, Thoma L, Miller DD. Emerging trends in oral delivery of peptide and protein drugs. Crit Rev Ther Drug Carrier Syst. 2003;20(2-3):153-214.
3. Hamman JH, Enslin GM, Kotzé AF. Oral delivery of peptide drugs: barriers and developments. Bio Drugs. 2005;19(3):165-177.

4. Wang M, Sun B, Feng J, et al. Investigation of transport mechanism of exendin-4 across Madin Darby canine kidney cell monolayers. Biol Pharm Bull. 2012;35(5):745-752.

5. Gedulin BR, Smith PA, Jodka CM, et al. Pharmacokinetics and pharmacodynamics of exenatide following alternate routes of administration. Int J Pharm. 2008;356(1-2):231-238.

6. Jin $\mathrm{CH}$, Chae SY, Son S, et al. A new orally available glucagon-like peptide-1 receptor agonist, biotinylated exendin-4, displays improved hypoglycemic effects in db/db mice. J Control Release. 2009;133(3): 172-177.

7. Gao M, Jin Y, Tong Y, Tian H, Gao X, Yao W. A site-specific PEGylated analog of exendin-4 with improved pharmacokinetics and pharmacodynamics in vivo. J Pharm Pharmacol. 2012;64(11):1646-1653.

8. Kwon KC, Nityanandam R, New JS, Daniell H. Oral delivery of bioencapsulated exendin-4 expressed in chloroplasts lowers blood glucose level in mice and stimulates insulin secretion in beta-TC6 cells. Plant Biotechnol J. 2013;11(1):77-86.

9. Nguyen HN, Wey SP, Juang JH, et al. The glucose-lowering potential of exendin-4 orally delivered via a $\mathrm{pH}$-sensitive nanoparticle vehicle and effects on subsequent insulin secretion in vivo. Biomaterials. 2011;32(10):2673-2682.

10. Bivas-Benita M, Romeijn S, Junginger HE, Borchard G. PLGA-PEI nanoparticles for gene delivery to pulmonary epithelium. Eur J Pharm Biopharm. 2004;58(1):1-6.

11. Tahara K, Furukawa S, Yamamoto H, Kawashima Y. Hybrid-modified poly(D,L-lactide-co-glycolide) nanospheres for a novel cellular drug delivery system. Int J Pharm. 2010;392(1-2):311-313.

12. Yang R, Shim WS, Cui FD, et al. Enhanced electrostatic interaction between chitosan-modified PLGA nanoparticle and tumor. Int J Pharm. 2009;371(1-2):142-147.

13. Yuan X, Shah BA, Kotadia NK, Li J, Gu H, Wu Z. The development and mechanism studies of cationic chitosan-modified biodegradable PLGA nanoparticles for efficient siRNA drug delivery. Pharm Res. 2010;27(7):1285-1295.

14. Schipper NG, Olsson S, Hoogstraate JA, deBoer AG, Vårum KM, Artursson P. Chitosans as absorption enhancers for poorly absorbable drugs 2: mechanism of absorption enhancement. Pharm Res. 1997; 14(7):923-929.

15. Ward PD, Tippin TK, Thakker DR. Enhancing paracellular permeability by modulating epithelial tight junctions. Pharm Sci Technolo Today. 2000;3(10):346-358.

16. Tahara K, Sakai T, Yamamoto H, Takeuchi H, Hirashima N, Kawashima Y. Improved cellular uptake of chitosan-modified PLGA nanospheres by A549 cells. Int J Pharm. 2009;382(1-2):198-204.

17. Tahara K, Sakai T, Yamamoto H, Takeuchi H, Kawashima Y. Establishing chitosan coated PLGA nanosphere platform loaded with wide variety of nucleic acid by complexation with cationic compound for gene delivery. Int J Pharm. 2008;354(1-2):210-216.

18. Chakravarthi SS, Robinson DH. Enhanced cellular association of paclitaxel delivered in chitosan-PLGA particles. Int J Pharm. 2011;409(1-2):111-120.

19. Murugeshu A, Astete C, Leonardi C, Morgan T, Sabliov CM. Chitosan/ PLGA particles for controlled release of alpha-tocopherol in the GI tract via oral administration. Nanomedicine (Lond). 2011;6(9):1513-1528.

20. Slütter B, Bal S, Keijzer C, et al. Nasal vaccination with N-trimethyl chitosan and PLGA based nanoparticles: nanoparticle characteristics determine quality and strength of the antibody response in mice against the encapsulated antigen. Vaccine. 2010;28(38):6282-6291.

21. Zhang X, Sun M, Zheng A, Cao D, Bi Y, Sun J. Preparation and characterization of insulin-loaded bioadhesive PLGA nanoparticles for oral administration. Eur J Pharm Sci. 2012;45(5):632-638.

22. Ungaro F, d'Angelo I, Coletta C, et al. Dry powders based on PLGA nanoparticles for pulmonary delivery of antibiotics: modulation of encapsulation efficiency, release rate and lung deposition pattern by hydrophilic polymers. J Control Release. 2012;157(1):149-159. 
23. Nafee N, Taetz S, Schneider M, Schaefer UF, Lehr CM. Chitosan-coated PLGA nanoparticles for DNA/RNA delivery: effect of the formulation parameters on complexation and transfection of antisense oligonucleotides. Nanomedicine. 2007;3(3):173-183.

24. Gaumet M, Gurny R, Delie F. Interaction of biodegradable nanoparticles with intestinal cells: the effect of surface hydrophilicity. Int J Pharm. 2010;390(1):45-52.

25. Guo C, Gemeinhart RA. Understanding the adsorption mechanism of chitosan onto poly(lactide-co-glycolide) particles. Eur J Pharm Biopharm. 2008;70(2):597-604.

26. Harush-Frenkel O, Rozentur E, Benita S, Altschuler Y. Surface charge of nanoparticles determines their endocytic and transcytotic pathway in polarized MDCK cells. Biomacromolecules. 2008;9(2):435-443.

27. Irvine JD, Takahashi L, Lockhart K, et al. MDCK (Madin-Darby canine kidney) cells: a tool for membrane permeability screening. J Pharm Sci. 1999;88(1):28-33.

28. Fujiwara T, Akita H, Harashima H. Intracellular fate of octaargininemodified liposomes in polarized MDCK cells. Int J Pharm. 2010; 386(1-2):122-130.

29. Win KY, Feng SS. Effects of particle size and surface coating on cellular uptake of polymeric nanoparticles for oral delivery of anticancer drugs. Biomaterials. 2005;26(15):2713-2722.

30. Rejman J, Oberle V, Zuhorn IS, Hoekstra D. Size-dependent internalization of particles via the pathways of clathrin- and caveolae-mediated endocytosis. Biochem J. 2004;377(Pt 1):159-169.
31. Khalil IA, Kogure K, Futaki S, Harashima H. High density of octaarginine stimulates macropinocytosis leading to efficient intracellular trafficking for gene expression. J Biol Chem. 2006;281(6): 3544-3551.

32. Dyer AM, Hinchcliffe M, Watts P, et al. Nasal delivery of insulin using novel chitosan based formulations: a comparative study in two animal models between simple chitosan formulations and chitosan nanoparticles. Pharm Res. 2002;19(7):998-1008.

33. Won YW, Lim KS, Kim YH. Intracellular organelle-targeted non-viral gene delivery systems. J Control Release. 2011;152(1):99-109.

34. Benfer M, Kissel T. Cellular uptake mechanism and knockdown activity of siRNA-loaded biodegradable DEAPA-PVA-g-PLGA nanoparticles. Eur J Pharm Biopharm. 2012;80(2):247-256.

35. Sahay G, Alakhova DY, Kabanov AV. Endocytosis of nanomedicines. J Control Release. 2010;145(3):182-195.

36. Reix N, Parat A, Seyfritz E, et al. In vitro uptake evaluation in Caco-2 cells and in vivo results in diabetic rats of insulin-loaded PLGA nanoparticles. Int J Pharm. 2012;437(1-2):213-220.

37. Panyam J, Zhou WZ, Prabha S, Sahoo SK, Labhasetwar V. Rapid endo-lysosomal escape of poly(DL-lactide-co-glycolide) nanoparticles: implications for drug and gene delivery. FASEB J. 2002;16(10): 1217-1226.

38. Bakhru SH, Altiok E, Highley C, et al. Enhanced cellular uptake and long-term retention of chitosan-modified iron-oxide nanoparticles for MRI-based cell tracking. Int J Nanomedicine. 2012;7:4613-4623.
International Journal of Nanomedicine

\section{Publish your work in this journal}

The International Journal of Nanomedicine is an international, peerreviewed journal focusing on the application of nanotechnology in diagnostics, therapeutics, and drug delivery systems throughout the biomedical field. This journal is indexed on PubMed Central, MedLine, CAS, SciSearch $\AA$, Current Contents ${ }^{\circledR} /$ Clinical Medicine,

\section{Dovepress}

Journal Citation Reports/Science Edition, EMBase, Scopus and the Elsevier Bibliographic databases. The manuscript management system is completely online and includes a very quick and fair peer-review system, which is all easy to use. Visit http://www.dovepress.com/ testimonials.php to read real quotes from published authors. 